\title{
Índices micrometeorológicos e internações de crianças por doenças respiratórias em Santarém, Oeste do Pará.
}

\author{
Micrometeorological index and children hospitalizations for respiratory diseases in Santarém, \\ Western Pará.
}

\author{
Ana Carla dos Santos Gomes ${ }^{1}$, Gabriel Brito Costaㄹ, Roseilson Souza do Vale ${ }^{3}$, Raoni Aquino \\ Silva de Santana ${ }^{4}$, Sarah Suely Alves Batalha ${ }^{5}$, Júlio Tóta da Silva ${ }^{6}$ e David Roy Fitzjarrald ${ }^{7}$ \\ 1,2,3,4,5,6 Universidade Federal do Oeste do Pará, PA, Brasil \\ anacarlasg02@gmail.com; gabrielbritocosta@gmail.com; roseilsondovale@gmail.com; raoniass@gmail.com; \\ ssa.batalha@gmail.com; totaju@gmail.com \\ ${ }^{7}$ University at Albany, State University of New York, Estados Unidos da América do Norte \\ dfitzjarrald@albany.edu
}

\begin{abstract}
Resumo
O objetivo deste estudo foi captar associações entre internações por doenças respiratórias e o índice micrometeorológico do município de Santarém, localizado na região do Oeste do Pará no ano de 2010. Utilizaram-se números de internações de crianças de 0 a 4 anos e dados de variáveis meteorológicas (umidade relativa do ar, temperatura do ar, precipitação pluvial, pressão atmosférica). Calculou-se os índices micrometeorológicos, por meio da análise de componente principal, cada componente principal é uma combinação linear de todas as variáveis originais, independentes entre si e estimadas com o propósito de reter, o máximo de informação da variação total contida nos dados; Para detectar a associação utilizou-se as equações de estimação generalizadas que são empregadas quando se deseja ajustar, modelos para dados longitudinais, foi considerado a distribuição de probabilidade Poisson e a estrutura para a matriz de correlação do tipo permutável. Os resultados sinalizaram que o maior número de internações ocorreu no mês de junho coincidindo com período de transição entre as estações chuvosa e seca. Foram observadas associações estatísticas significativas, ressaltando que foi captado o risco relativo de $10 \%$ devido à sinergia das variáveis meteorológicas. Espera-se assim auxiliar no planejamento de políticas públicas ambientais e de saúde.
\end{abstract}

Palavras-chave: Análise de Componente Principal. Microclima. Amazônia. Saúde.

\begin{abstract}
The aim of this study was to attract associations between hospitalizations for respiratory diseases and micrometeorological index in the Santarém city, located in the West Pará region in the year 2010. It was used numbers of children hospitalizations with 0-4 years old and weather data variables (relative humidity, air temperature, precipitation, atmospheric pressure). Was calculated the Micrometeorological index, through principal component analysis, were each principal component is a linear combination of all the original variables, independent of each other and estimated in proposal to retain the maximum total variation information contained in the data; To detect the association we used generalized estimating equations that are used when you want to fit models for longitudinal data. The results suggest that the greatest number of admissions occurred in June coinciding with the transition period between the rainy and dry seasons. Statistically significant associations were observed, noting that the relative risk of $10 \%$ was captured for the increase in hospitalizations due to synergy of meteorological variables. This is expected to assist in the planning of public policies and environmental health.
\end{abstract}

Keywords: Principal Component Analysis. Microclimate. Amazon. Health. 


\section{Introdução}

A relação existente entre os impactos adversos das condições climáticas na saúde não apresenta uma associação simples. Uma vez que a interação entre a exposição a um ou mais fatores de risco e o desenvolvimento de uma doença não é reconhecida facilmente. Especialmente quando se presume que a relação se dê também a partir de comportamentos sociais comuns, como o tipo de alimentação (GOMES et al., 2013).

Sabe-se que o efeito das condições atmosféricas no homem pode ser imediato quando se trata das doenças agudas; nas doenças crônicas, as primeiras manifestações podem surgir após muitos anos de exposição aos fatores de risco de forma única ou contínua, por exemplo, radiações ionizantes e radiação solar. Os fatores de risco podem ser encontrados no ambiente físico, hereditários ou representar hábitos próprios de um determinado ambiente social e cultural (MERRIL, 2008).

Segundo Chagas e Marques (2007) os efeitos meteoro-trópicos tendem a ocorrer de forma sincrônica em grande território, sob a influência das mesmas condições de tempo o que afeta significativamente a população local.

Biologicamente, essa influência é justificada para que o organismo consiga se adaptar ao meio. A temperatura central do organismo humano é regulada pelo hipotálamo e a qualquer alteração térmica, a temperatura do corpo ativa mecanismos internos de compensação. Se for preciso baixar a temperatura interna, o hipotálamo promove vaso dilatação e sudorese, transferindo esse calor para o meio externo. Embora o corpo se adapte ao meio, seja ele quente ou frio, os limites de temperatura interferem consideravelmente no bem estar das pessoas (KELLOGG, 2006).

Bakonyi et al., 2004 e Gouveia et al., 2004 afirmam que os grupos mais suscetíveis as alterações climáticas são crianças e idosos. Em relação às doenças respiratórias pode-se destacar o fato de que em crianças, de maneira geral, a sensibilidade a temperaturas está diretamente associada à imaturidade de seu aparelho respiratório, principalmente nos três primeiros anos de vida, quando novos alvéolos se desenvolvem e após esta idade quando ocorre a expansão do crescimento pulmonar (GAUDERMAN, 2004). Diante do mencionado, o objetivo deste estudo foi captar associações entre internações por doenças respiratórias e o índice micrometeorológico do município de Santarém, localizado na região do Oeste do Pará no ano de 2010. A justificativa para a escolha desse período se dá devido estudos (Lewis et al., 2011; Marengo et al., 2011) apontarem este ano sendo o que apresentou a seca mais drástica na Amazônia nos últimos 100 anos, o que provavelmente influenciou no aumento da temperatura do ar da região.

\section{Material e Métodos}

A área de estudo foi o município de Santarém-PA, localizado no Oeste do Pará, região pertencente à Amazônia Central. Trabalhou-se com dados mensais de precipitação pluvial, pressão atmosférica, temperatura e umidade relativa do ar coletados pela Rede de Meteorologia da Aeronáutica (Redemet) do Aeroporto Internacional de Santarém - Maestro Wilson Fonseca. Os registros de internações de crianças de 0 a 4 anos por doenças respiratórias (Asma, Bronquite, Bronquiolite, Laringite, Pneumonia, Sinusite e Infecções agudas das vias aéreas superiores), segundo o local de residência, foram disponibilizados pelo Banco de Dados do Sistema Único de Saúde (DATASUS) do Ministério da Saúde, no período de Janeiro a Dezembro de 2010, totalizando o período de um ano.

Os índices micrometeorológicos mensais foram construídos por meio da técnica da estatística multivariada, analise de componentes principais (ACP) que consiste em transformar um conjunto de variáveis originais em outro conjunto de variáveis de mesma dimensão (KHATTREE e NAIK, 2000). Cada componente principal é uma combinação linear de todas as variáveis originais, são independentes entre si e estimadas com o propósito de reter, em ordem de estimação, o máximo de informação, em 
termos da variação total contida nos dados. Procura-se redistribuir a variação observada nos eixos originais de forma a se obter um conjunto de eixos ortogonais não correlacionados. Esta técnica é apropriada para geração de índices e agrupamento de indivíduos ou variáveis (JOHNSON e WICHERN, 1999).

Para verificar as associações utilizaram-se as Equações de Estimação Generalizadas propostas por Zeger e Liang (1986) e Liang e Zeger (1986).

Considera-se $y_{i}$, o vetor de variável resposta, obtidos pelas internações de crianças por doenças respiratórias de dimensão $t_{i} \times 1$, isto é, $\boldsymbol{y}_{i}=\left(\mathrm{y}_{\mathrm{i} 1}, \mathrm{y}_{\mathrm{i} 2}, \ldots, \mathrm{y}_{\mathrm{iti}}\right)^{\prime}$, e seja $\mathrm{X}_{\mathrm{i}}=\left(\mathrm{x}_{\mathrm{i} 1}, \mathrm{x}_{\mathrm{i} 2}, \ldots\right.$, $\left.\mathrm{X}_{\mathrm{iti}}\right)^{\prime}$ a matriz de covariáveis, de dimensão $t_{\mathrm{i}} \mathrm{x}$ $p$, cujas linhas são os vetores $x_{i t}=\left(x_{i t 1}, \ldots, x_{i t p}\right)^{\prime}$, para o i-ésima unidade observacional $(i=1,2, \ldots$, n) nos t-ésimos instantes de observações (tempos), $t=1,2, \ldots, t_{\mathrm{i}}$. Assume-se, a princípio, que a distribuição marginal da variável aleatória $Y_{\text {it }}$ é conhecida e pertence à família definida em (1), ou seja:

$$
f\left(y_{i t}, \theta_{i t}, \phi\right)=\exp \left\{\frac{1}{a_{i}(\phi)}\left[y_{i t}-b\left(\phi_{i t}\right)\right]+c\left(y_{i t}, \phi\right)\right\}
$$

na qual a inclusão do índice $t$ é para considerar as observações repetidas no tempo. Assume-se também que $E\left(Y_{i t}\right)=\mu_{i t}=b^{\prime}\left(\theta_{i t}\right)$, $\operatorname{Var}\left(Y_{\mathrm{it}}\right)=\mathrm{ai}(\phi) \mathrm{b}^{\prime \prime}$

$\left(\phi_{i t}\right)=a_{i}(\phi) V\left(\mu_{i t}\right)$, em que $V\left(\mu_{i t}\right)$ é a função de variância. Também, em grande parte dos casos, ai $(\phi)=\phi$. Pode-se, então, definir um modelo linear generalizado para cada instante de tempo $t$, acrescentando uma função que faz a ligação entre a parte aleatória (1) e a parte sistemática: $\eta_{\mathrm{it}}=\mathrm{g}\left(\mu_{\mathrm{it}}\right)$, em que $\eta_{\mathrm{it}}=\mathrm{x}^{\prime}{ }_{\mathrm{it}} \beta$ é o preditor linear, $\beta=\left(\beta_{1}, \beta_{2}, \ldots, \beta_{p}\right)^{\prime}$ é o vetor de parâmetros desconhecidos e de interesse e $\mathrm{g}($.) é a função de ligação.

Para o estudo em questão, considerou-se a distribuição de probabilidade Poisson, em que $\eta$ it $=\ln (\mu \mathrm{it})=\beta 0+\beta 1 X$, cuja interpretação da exponencial do parâmetro estimado representa o risco relativo.

As estimações originadas pelas GEE são realizadas pelo método de QuaseVerossimilhança, proposto por Wedderburn (1974), o qual requer apenas especificação de uma função de variância para a variável resposta e a relação funcional entre a resposta média e os parâmetros $\beta$. A estrutura de correlação utilizada foi do tipo permutável (exchangeable) na qual se considera que a correlação entre as observações das variáveis de um mesmo grupo é a mesma. Essa é a estrutura de correlação assumida em um modelo de efeitos aleatórios com um intercepto aleatório para cada variável (LAIRD; WARE, 1982). Os métodos estatísticos utilizados foram realizados com o auxílio do software estatístico livre R 2.15.0.

\section{Resultados e Discussões}

Na Figura 1 observa-se a variabilidade mensal das variáveis meteorológicas ao longo do ano de 2010. Os maiores valores médios ocorreram em outubro $\left(33,29{ }^{\circ} \mathrm{C}\right)$ para a temperatura do ar, maio para a precipitação $(260,5 \mathrm{~mm})$, abril para a umidade relativa $(99,23 \%)$ e junho para a pressão atmosférica $(993,33 \mathrm{hPa})$. O mês de junho pode ser considerado o período de transição entre as estações chuvosa (dezembro a maio) e seca (julho a novembro).
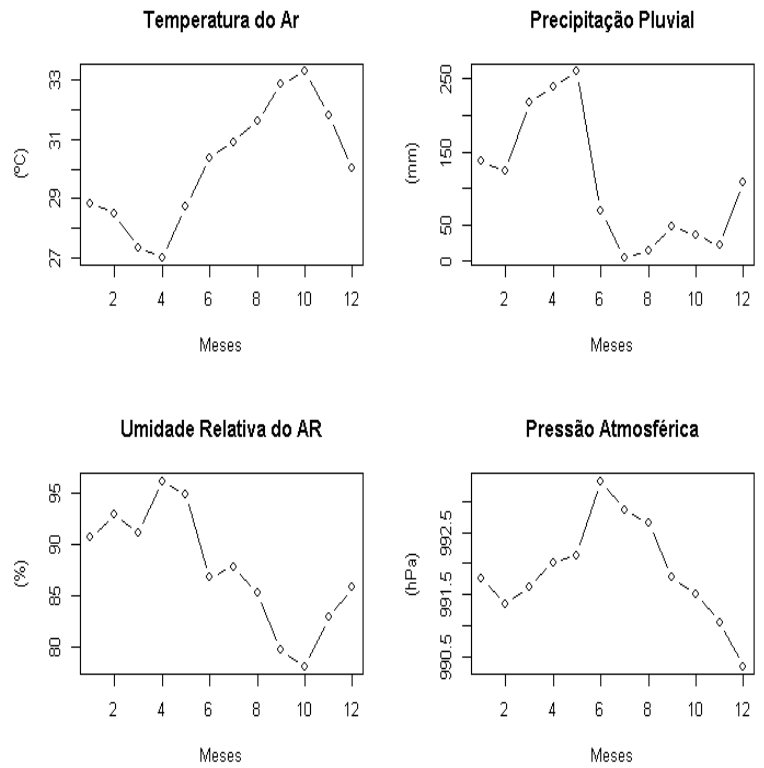

Figura 1 - Variabilidade das variáveis meteorológicas em Santarém-PA em 2010

Constatou-se a partir da Figura 2 que o maior número de internações de crianças por causas respiratórias em 2010 ocorreu no mês de junho (período de transição entre as estações). O qual é caracterizado por altas temperaturas, diminuição das chuvas e da 
umidade relativa e aumento da pressão atmosférica.

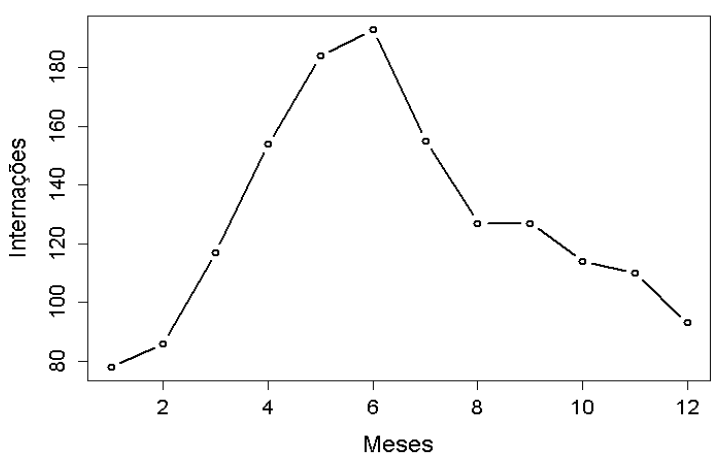

Figura 2 - Variabilidade das internações de crianças por doenças respiratórias em SantarémPA em 2010

Para a construção do índice micrometeorológicos da região de Santarém, foi utilizado informações de todas as variáveis meteorológicas em estudo conforme foi observado anteriormente na Figura 1. É possível verificar na Tabela 1, o desvio padrão, a proporção da variância total e a proporção acumulada contida em cada componente. Nota-se que até a $3{ }^{\underline{a}}$ componente já é possível explicar $98 \%$ da variação existente nos dados.

Tabela 1 -Variação existente nos dados; desvio padrão, proporção da variância total e proporção acumulada na construção do índice micrometeorológico de Santarém em 2010

\begin{tabular}{lcccc}
\hline & $1^{\mathrm{a}}$ Comp. & 2 $^{\mathrm{a}}$ Comp. & 3Comp. $^{\mathrm{a}}$ & $4^{\mathrm{a}}$ Comp. \\
\hline $\begin{array}{l}\text { Desvio- } \\
\text { padrão }\end{array}$ & 1,652 & 1,022 & 0,4257 & 0.2142 \\
$\begin{array}{l}\text { Proporção } \\
\text { da variância }\end{array}$ & 0,682 & 0,261 & 0,0453 & 0.0115 \\
$\begin{array}{l}\text { Proporção } \\
\text { cumulativa }\end{array}$ & 0,682 & 0,943 & 0,9885 & 1.0000 \\
\hline
\end{tabular}

Os pesos sugeridos após a ACP podem ser observados na Tabela 2. A $1^{\text {a }}$ componente contém informações da precipitação, temperatura e umidade com pesos similares. No entanto na $2^{\mathrm{a}}$ componente a pressão atmosférica foi quem mais contribuiu, na $3^{\mathrm{a}}$ foi a precipitação e na $4^{\mathrm{a}}$ a temperatura.
Tabela 2 - Pesos das variáveis meteorológicas em cada componente principal

\begin{tabular}{lcccc}
\hline & $1^{\text {a }}$ Comp. & $2^{\text {a }}$ Comp. & 3 Comp. & $4^{\text {a }}$ Comp. \\
\hline Prec. $(\mathrm{mm})$ & 0,560 & 0,150 & 0,812 & -- \\
Temp. $\left({ }^{\circ} \mathrm{C}\right)$ & $-0,591$ & -- & 0,357 & $-0,723$ \\
UR(\%) & 0,580 & $-0,166$ & $-0,418$ & $-0,679$ \\
Pres.(hPa) & -- & $-0,974$ & 0,193 & 0,114 \\
\hline
\end{tabular}

Exceto com a $4^{\text {a }}$ componente, é possível constatar associações significativas com 95\% de confiabilidade entre as internações de idosos por doenças cardiovasculares e as componentes principais (Tabela 3). Observase que é a $3^{\mathrm{a}}$ componente que contém informações de todas as variáveis meteorológicas, logo é ela também que informa, por meio do risco relativo, que as condições micrometeorológicas influenciaram no aumento em $10 \%$ do número de internações de crianças por doenças respiratórias no ano de 2010.

Tabela 3 - Modelo das Equações de Estimação Generalizada das Doenças Respiratórias de Crianças e o Índice Micrometeorológico de Santarém em 2010

\begin{tabular}{cccc}
\hline & Estimativa & Erro Padrão & P-valor \\
\hline Intercepto & $-292,6712$ & 56,4113 & $<0,001$ \\
$1^{\text {a }}$ Comp. & $-0,0579$ & 0,0156 & $<0,001$ \\
$2^{2}$ Comp. & $-0,2901$ & 0,0549 & $<0,001$ \\
$3^{\text {a }}$ Comp. & 0,0934 & 0,0162 & $<0,001$ \\
$4^{\text {a }}$ Comp. & $-0,0463$ & 0,0299 & 0,1215 \\
\hline
\end{tabular}

$5 \%$ de significância.

$\mathrm{O}$ índice micrometeorológico do município de Santarém no ano de 2010 é dado pela $3^{3}$ componente principal, apresentando a seguinte fórmula:

$$
\begin{gathered}
\mathrm{I}_{\mathrm{M}}=0,812 \cdot \text { Prec }+0,357 \cdot \text { Temp }-0,418 \cdot \mathrm{Ur}+ \\
\text { 0,193.Pres. }
\end{gathered}
$$

Sendo a precipitação a variável com maior peso, logo a que mais está associada às internações de crianças por doenças respiratórias em Santarém no ano de 2010, seguida pela umidade relativa do ar, porém de forma inversa, ou seja, a diminuição dos 
valores da umidade relativa contribui para o aumento das internações. Estes resultados corroboram com os encontrados por Duarte e Mascarenhas (2007) e Rosa et al., (2008).

Gandu et al., (2004) realizaram simulações para a região leste da Amazônia e sugerem que nas áreas costeiras do Pará e sobre os grandes rios da região, o desmatamento pode ser responsável por um decréscimo de precipitação. Por Santarém se localizar as margens do rio Tapajós sua taxa de precipitação pode ter sido diminuída o que pode ter contribuindo para o aumento das internações por doenças respiratórias.

\section{Conclusões}

Com base nas investigações realizadas, observou-se o maior número de internações de crianças por doenças respiratórias em 2010 no mês de junho, coincidindo com mês de transição entre as estações chuvosa e seca. Constatou-se associação estatística significativa entre o índice e as internações, ressaltando que a sinergia entre as variáveis meteorológicas, representadas pelo índice micrometeorológico, influencia no aumento em $10 \%$ do número de internações no período estudado. Por fim destaca-se que dentre as varáveis meteorológicas a precipitação foi a que teve o maior peso na construção do índice.

\section{Agradecimentos}

Agradecemos a Universidade Federal do Oeste do Pará pelo incentivo e apoio à pesquisa e à Coordenação de Aperfeiçoamento de Pessoal de Nível Superior (CAPES) pela ajuda de custo para o desenvolvimento desta pesquisa.

\section{Referências}

AGRESTI, A. (1996). An Introduction to categorical data analysis. John Wiley and Sons. Inc. New York.

BAKONYI, C. S. M., DANNI-OLIVEIRA, M.I., MARTINS, L. C. (2004). Poluição atmosférica e doenças respiratórias em crianças na cidade de Curitiba, PR. Rev. Saúde Pública, v.38, n. 5, p.695-700. out.

CHAGAS, C. MARQUES. F. (2007). Novo índice avalia relações entre clima e saúde. In: Revista Manguinhos. Clima e Saúde - p. 1823.

DUARTE, A. F., MARCARENHAS, M. D. M. (2007). Manifestações do bioclima do acre sobre a saúde humana no contexto socioeconômico da Amazônia. Amazônia: Ci. \& Desenv., Belém, v. 3, n. 5, jul./dez.

GANDU, A. W., COHEN, J. C. P., SOUZA, J. R. S DE. (2004). Simulations of deforestation in Eastern Amazon using a higher-resolution model. Theoretical and Applied Climatology, 78: 123-135

GOMES, A. C. DOS S., LUCIO, P. S., SPYRIDES, M. H. C. (2013). Influence of pollution from particulate matter at the hospitalizations of asthmatic children in area of Great São Paulo. Revista Brasileira de Geografia Física, Vol. 6, No 4 (2013)

GOUVEIA, N., BREMNER, S.A., NOVAES, H. M., (2004). Association between ambient air pollution and birth weight in São Paulo, Brazil. J Epidemiol Community Health; 58:117.

LAIRD, N. M., WARE, J. H. (1982). Randomeffects models for longitudinal data. Biometrics, v.38:963-974.

LEWIS, S. L., P. M. BRANDO. O. L., PHILLIPS. G. M. F., Van Der Heijden. and Nepstad, D. (2011). The 2010 Amazon drought. Science. $331 . \quad 554$. doi:10.1126/science.1200807. 
LIANG, K. Y., ZEGER, S. L. (1986). Longitudinal data analysis using generalized linear models. Biometrika. 73:13-22.

KELLOGG, D. L. (2006). In vivo mechanisms of cutaneous vasodilation and vasoconstriction in humans during thermoregulatory challenges. J Appl Physiol. 100:1709-18

MARENGO, J. A., J. TOMASELLA. L. M., ALVES. W. R., SOARES. AND D. A. RODRIGUEZ (2011). The drought of 2010 in the context of historical droughts in the Amazon region. Geophys. Res. Lett.. 38. L12703. doi:10.1029/2011GL047436.

MERRIL, R. M. (ed). (2008). Environmental epidemiology: principles and methods. Massachusetts: Jones and Bartlett Publishers.

R DEVELOPMENT CORE TEAM (2009). R: A language and environment for statistical computing. $\mathrm{R}$ Foundation for Statistical Computing. Vienna. Austria.

ROSA, A. M., IGNOTTI, E., HACON, S.S., CASTRO, H.A. (2008). Análise das internações por doenças respiratórias em Tangará da Serra - Amazônia Brasileira. J Bras Pneumol. 34:575-82.

WEDDERBURN, RWM. 1974. Quasilikelihood functions, generalized linear models, and the Gauss-Newton method. Biometrika. 61:439-447.

ZEGER, S. L., LIANG, K. Y. (1986). Longitudinal data analysis for discrete and continuous outcomes. Biometrics. 42:1(3)121130. 